\title{
A Novel Approach of Targeted Immunotherapy against Adenocarcinoma Cells with Nanoparticles Modified by CD16 and MUC1 Aptamers
}

\author{
Lianyuan Yu, Yan Hu, Jinhong Duan, and Xian-Da Yang \\ Institute of Basic Medical Sciences, Chinese Academy of Medical Sciences, School of Basic Medicine of Peking Union Medical College, \\ Beijing 100005, China
}

Correspondence should be addressed to Xian-Da Yang; ayangmd@gmail.com

Received 14 April 2015; Accepted 4 June 2015

Academic Editor: Tae-Yub Kwon

Copyright $\odot 2015$ Lianyuan Yu et al. This is an open access article distributed under the Creative Commons Attribution License, which permits unrestricted use, distribution, and reproduction in any medium, provided the original work is properly cited.

\begin{abstract}
Mucin 1 (MUC1) is a potentially important target of cancer therapy, being a glycoprotein that is overexpressed on cell surface of many types of adenocarcinomas, including breast, ovarian, colon, lung, and prostatic cancers. Several MUC1-targeted drug delivery systems have been developed and reported, but mobilizing natural killer cells (NK) to fight against MUC1-positive tumor has not been attempted. In this study, we introduced a novel amphipathic nanoparticle (NP) for enhancing the NK cytotoxicity to MUC1-positive cancer cells. The amphipathic NP had CD16 and MUC1 aptamers on its surface and was designed to bind with both the CD16-positive NK cells and the MUC1-positive tumor cells and pull the two types of cells close to each other. The fabricated amphipathic NP had an average size of $574 \mathrm{~nm}$. The aptamers' conjugation with the NP was confirmed by DNA hybridization experiments. Dark-field microscopy revealed that the amphipathic NP could recruit more NK cells to the vicinity of MUC1-positive cancer cells. Additionally, the amphipathic NP significantly enhanced the NK cytotoxicity to MUC1-positive cancer cells $(p<0.01)$, but not that to the MUC1-negative control cells. The results suggest that NK cells may potentially be mobilized to selectively fight against MUC1-positive cancer cells.
\end{abstract}

\section{Introduction}

Traditional cancer therapies include surgery, chemotherapy, and radiotherapy, but these therapies have their own limitation or drawbacks as in clinical application. Surgery is quite effective for early treatment of solid tumors, but it is useless for advanced tumor, nonsolid tumor, and leukemia. As in chemotherapy, long-term use of chemotherapy drugs will develop the drug resistance of tumor cells and reduce the efficacy of these drugs. Beyond that, as well as eliminating cancer cells, the chemotherapy drugs also attack the normal tissue and immune system of cancer patients, which will result in drug toxicity [1]. Additionally, general and local radiotherapy may cause seriously adverse effect, such as osteoradionecrosis, radiation pneumonitis, and systemic reactions [2]. Besides the above cancer therapies, immunotherapy is a type of tumor therapy built on the principle of stimulating the patient's immune system to attack and eliminate tumor cells [3]. The currently existing immunotherapies mainly include cell-based therapies, antibody therapies, and cytokine therapies $[4,5]$. Compared with chemotherapy and radiotherapy, immunotherapy has several advantages, including low toxicity and long-acting in vivo response against tumor cells $[6,7]$. Because most grown tumors have immune escape mechanisms, the major challenge facing most immunotherapies is to induce an effective immune response that is strong and durable enough to fight against the cancer cells [8]. As a result, it is necessary to explore new approaches for promoting targeted immune response against cancer cells.

In this study, we developed a novel nanoparticle modified by CD16 and MUC1 aptamers $[9,10]$, in order to gather NK lymphocytes and adenocarcinoma cells together and enhance the NK reaction against these cancer cells. CD16, namely, Fcy receptor III, is mainly expressed on the surface of NK cells, 
monocytes, macrophages, and polymorphonuclear cells. MUC1 mucin, a glycoprotein, is underexpressed on normal tissue but gets overexpressed on the cell surface of most adenomas, including colon, breast, ovarian, lung, and pancreatic cancers. Therefore, MUC1 protein is considered an ideal molecular target for immunotherapy [11]. Because the amphipathic NP has CD16 and MUC1 aptamers on its surface, theoretically it can bind with both NK and adenocarcinoma cells, pulling the two types of cells together. In this study, the targeting ligands employed are aptamers. As targeting ligands, aptamer has several advantages compared with antibody, including low immunogenicity, low preparation cost, and easy modification for various applications. Previously studies have reported aptamer as targeting agent to enhance drug delivery to MUC1-positive tumors [10] or probes for lymphoma cells [12]. Therefore, we chose aptamers as the targeting ligands to modify NP in this study. We now report that the aptamers modified amphipathic NP can pull together NK and MUC1-positive tumor cells and enhance NK cytotoxicity to these adenocarcinoma cells.

\section{Methods}

2.1. Cell Lines and Cultures. Cell lines of A549 (human lung cancer cell) and MD-MBA-231 (human breast cancer cell) were obtained from the Cell Resource Center of Chinese Academy of Medical Sciences (Beijing, China). Peripheral blood mononuclear cell (PBMC) used in our experiments was gathered through separation of venous blood from healthy human donors. The blood was firstly diluted with D-Hanks at equal volume. Lymphocyte separation medium (TBD, China) was decanted into the mixture and four layers appeared after centrifugation of $20 \mathrm{~min}$. The second layer was extracted by pipette and washed two times with D-Hanks to obtain PBMC. A549 and MD-MBA-231 cells were incubated in DMEM medium, which was supplemented with $100 \mathrm{u} / \mathrm{mL}$ penicillin, $100 \mathrm{mg} / \mathrm{mL}$ streptomycin, and 10\% fetal calf serum (Gibco). All cells were cultured in $5 \% \mathrm{CO}_{2}$ at $37^{\circ} \mathrm{C}$.

2.2. Construction and Characterization of Amphipathic NP. A previously identified MUC1 aptamer with the sequence of 5'-biotin-AACCGCCCAAATCCCTAAGAGTCGGACTGCAACCTATGCTATCGTTGATGTCTGTCCAAGCAACACAGACACACTACACACGCACA-3' was synthesized (Sangon Biotech, Shanghai, China). A previously selected CD16 aptamer with the sequence of $5^{\prime}$-biotinCCATTGCGGGGGTCTATACGTGAG GAAGAAGTGG-3' was also synthesized (Sangon Biotech, Shanghai, China). Five pmol MUC1 aptamer and 5 pmol CD16 aptamer were added to $20 \mu \mathrm{L}$ of streptavidin-coated magnetic beads (Promega, Germany) and fully mixed for $15 \mathrm{~min}$. The beads were washed thrice with $200 \mu \mathrm{L}$ PBS to remove unbound oligonucleotides. To measure the size distribution of the amphipathic NP, $10 \mu \mathrm{L}$ suspended NP was diluted into $1 \mathrm{~mL}$ PBS and analyzed with a dynamic light scattering (DLS) instrument (Zetasizer Nano ZS90, Malvern Instruments, Malvern, UK). Amphipathic NP samples were also evaluated by confocal microscopy (FV1000MPE, Olympus, USA).
2.3. Flow Cytometric Analysis. The binding of the aptamers to the target cells was evaluated by flow cytometry. FAMlabeled MUC1 and CD16 aptamers were synthesized (Sangon Biotech, Shanghai, China). Another aptamer that specifically binds with human epidermal growth factor receptor2 (HER2) was also synthesized to serve as a control. FAMlabeled MUC1 aptamer was incubated with A549 and MDMBA-231 cells for $30 \mathrm{~min}$. FAM-labeled HER2 aptamer was incubated with A549 cells. FAM-labeled CD16 aptamer was incubated with PBMCs. All the above cells were washed with $200 \mu \mathrm{L}$ PBS for three times and subjected to flow cytometry analysis (Accuri C6, USA).

To study whether the MUC1 and the CD16 aptamers were conjugated to the NP via the biotin-streptavidin reaction, DNA hybridization experiments were performed. MUC1 aptamer-modified NP, CD16 aptamer-modified NP, and MUC1CD16 aptamers-modified NP were constructed by mixing streptavidin-coated NPs (Promega, Germany) with MUC1 aptamer, CD16 aptamer, and a mixture of both aptamers (at 1:1 molar ratio) for $15 \mathrm{~min}$, respectively. Hybridizing DNA probes complementary to MUC1 or CD16 aptamers were incubated with the three types of particles for $30 \mathrm{~min}$. After washing for three times with $200 \mu \mathrm{L}$ PBS, the beads were subjected to flow cytometry analysis. All aptamers were biotinylated and all DNA probes were FAM-labeled.

For assessing the influence of aptamer-modification on the affinity of NP to target cells, competition blocking assays were conducted. Twenty thousand A549 cells were incubated with 40 pmol FAM-labeled MUC1 aptamer. Several items, including blank NP, MUC1 aptamer-modified NP, and unlabeled MUC1 aptamer, were separately added to the cells to compete with the FAM-labeled MUC1 aptamer. The mixtures were incubated for $30 \mathrm{~min}$, washed for three times with $200 \mu \mathrm{L}$ PBS, and subjected to flow cytometry analysis. Similar experiments were also conducted for CD16 and HER aptamers. Briefly, PBMC was incubated with FAM-labeled CD16 aptamer, while blank NP, CD16 aptamer-modified NP, and unlabeled CD16 aptamer were separately added to serve as competitive items. Moreover, HER2 aptamer-modified NP was also evaluated as a competitor of FAM-labeled MUC1 aptamer for binding with A549 cells.

2.4. Phase-Contrast Microscopy. The spatial relationship between live NK and A549 cells was studied with dark-field phase-contrast microscopy. Two groups of A549 cells were cultured in 6-well plate overnight and then cocultured with PBMC for 30 min. Amphipathic NP or blank NP were added to the two groups of cells. After washing with $1 \mathrm{~mL}$ PBS thrice, the cells were evaluated by phase-contrast microscopy.

2.5. In Vitro Cytotoxicity Assays. To evaluate whether the amphipathic NP would affect the NK reaction against A549 and MD-MBA-231 cells, NK cytotoxicity study was conducted. PBMC and A549 cells were cocultured in 96-well plates at effector : target ratio $(E: T)$ of $100: 1$. Cellular mixtures were treated separately with various items, including the amphipathic NP (MUC1-NP-CD16), unmodified NP, a mixture of free CD16 and MUC1 aptamers, and the NP modified 
by HER2 and CD16 aptamers (HER2-NP-CD16) for 10 hours at $37^{\circ} \mathrm{C}$. The nonadherent lymphocytes were washed off with $200 \mu \mathrm{L}$ PBS for three times. MTS assay was applied to evaluate the viability of the A549 cells, according to the standard protocol as outlined by the manufacturer (Promega, Germany). Similar experiments were also conducted on MD-MBA-231 cells. The PBMCs and MD-MBA-231 cells were cocultured at an $E: T$ ratio of $100: 1$ for $10 \mathrm{~h}$ at $37^{\circ} \mathrm{C}$ with the amphipathic NP. After washing the cells with $200 \mu \mathrm{L}$ PBS for three times, MTS assay was performed to assess the viability of the MDMBA-231 cells.

\section{Results}

3.1. Design of the Amphipathic NP. The primary function of the amphipathic NP designed in this study was to bring together the NK and the MUCl-positive tumor cells. To implement this goal, we fabricated an NP that was modified with both the CD16 and the MUC1 aptamers. The aptamers were connected with streptavidin-covered nanoparticle via the biotin-streptavidin reaction. Because the amphipathic NP had both CD16 and MUC1 aptamers on its surface, it theoretically should combine with both the NK and the MUC1positive cancer cells and bring them together. The overall design of the amphipathic NP was illustrated in Figure 1.

3.2. Verification of the Binding Property of the Aptamers. The capability of the aptamers to bind with their target cells was critical for the function of the amphipathic NP. Hence, we verified the binding properties of the aptamers against the target cells here in this study. FAM-labeled MUC1-aptamer was separately incubated with MUC1-positive A549 and MUC1-negative MD-MBA-231 cells, which were analyzed by flow cytometry. As shown in Figures 2(a) and 2(b), the MUC1 aptamer bound with the A549 but not the MD-MBA-231 cells, suggesting that the aptamer could recognize the MUC1positive A549 cells. This study also required a control aptamer that did not bind with MUC1-positive cells, for which we employed an aptamer that was originally designed to recognize the HER2 protein. FAM-labeled HER2 aptamer was incubated with MUC1-positive A549 cells, which was analyzed by flow cytometry. The results showed that the HER2 aptamer did not bind with the A549 cells (Figure 2(c)), indicating that it might serve as a control aptamer for this study.

The capability of the CD16 aptamer for binding with immune cells was also evaluated in this study. Because PBMC was rich in CD16-positive immune cells, here we assessed the affinity of CD16 aptamer to PBMC, which was commonly used as the experiment model in NK-related studies [9]. FAM-labeled CD16 aptamer was incubated with PBMC, which was subsequently analyzed by flow cytometry. As illustrated in Figure 2(d), CD16 aptamer generated a strong binding to PBMC, suggesting that the aptamer could recognize the CD16-positive immune cells.

3.3. Conjugation of Aptamers with Nanoparticles. In order for the amphipathic NP to bring together the NK and the MUC1positive cells, both the CD16 and the MUCl aptamers must

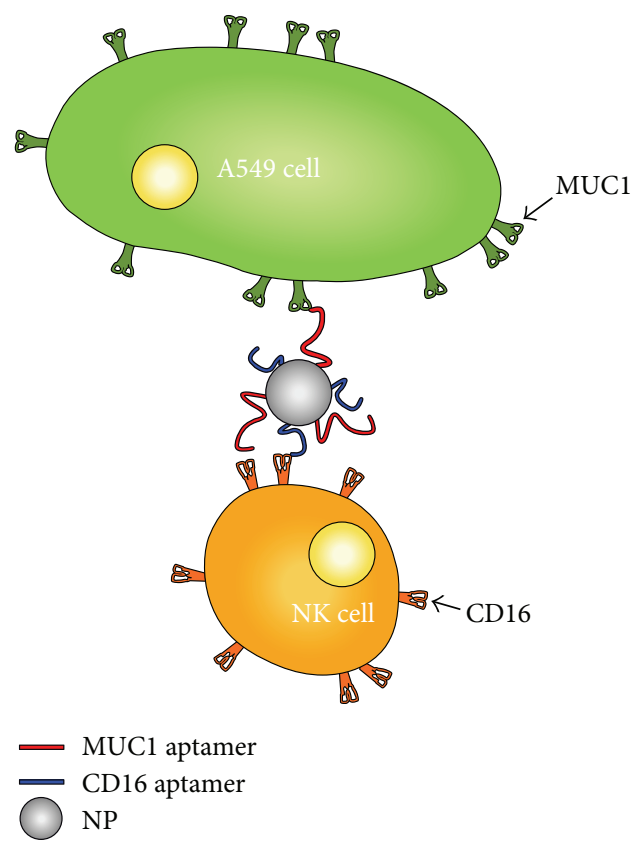

FIgURE 1: Illustration of the general design of the amphipathic NP. Biotinylated CD16 and MUC1 aptamers were connected with streptavidin-coated NP via biotin-streptavidin reaction. The proposed function of the amphipathic NP is to bring the NK to the vicinity of MUC1-positive cancer cell and induce an anticancer reaction.

be connected to the nanoparticle. To investigate whether the aptamers were indeed connected to the particle, DNA hybridization experiments were conducted. Two types of DNA probes (CD16 probe and MUC1 probe) were designed to complementarily recognize the CD16 and the MUC1 aptamers, respectively. The probes were separately incubated with NP, CD16 aptamer-modified NP (CD16-NP), MUC1 aptamermodified NP (MUC1-NP), and the amphipathic NP (CD16NP-MUC1), which were subjected to flow cytometry. As presented in Figure 3, CD16 aptamer-modified particles were positively stained by the CD16 probe, and MUC1-aptamer modified particles were stained by the MUC1 probe, while the amphipathic particles were positively stained by both probes. The results suggested that CD16 and MUC1 aptamers were indeed functionalized onto the surface of the amphipathic nanoparticles.

3.4. Morphological Characterization of the Amphipathic NPs. To investigate whether aptamer modification would affect the morphology and size of the iron NPs, blank NPs and aptamermodified NPs were analyzed by confocal microscopy and dynamic light scattering (DLS) assay. As shown in Figure 4, confocal microscopy revealed that aptamer-modified NPs had similar morphology as unmodified NPs. DLS assay revealed that both the unmodified and the aptamer-modified NPs had single-peak size distribution. The average size of the unmodified NPs was $491 \mathrm{~nm}$, whereas that of the aptamermodified NPs was $574 \mathrm{~nm}$, presumably because aptamermodification increased the nanoparticles' average diameter as measured by DLS. 


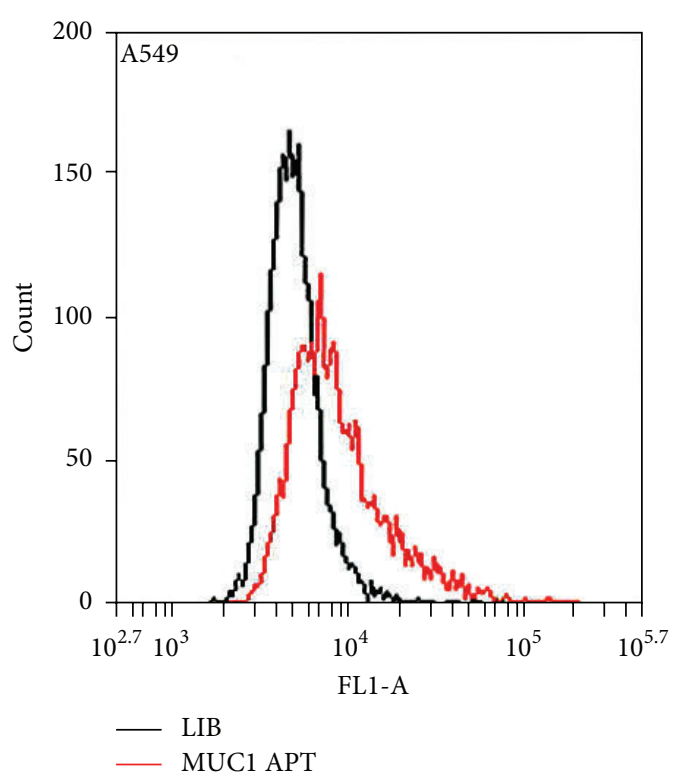

(a)

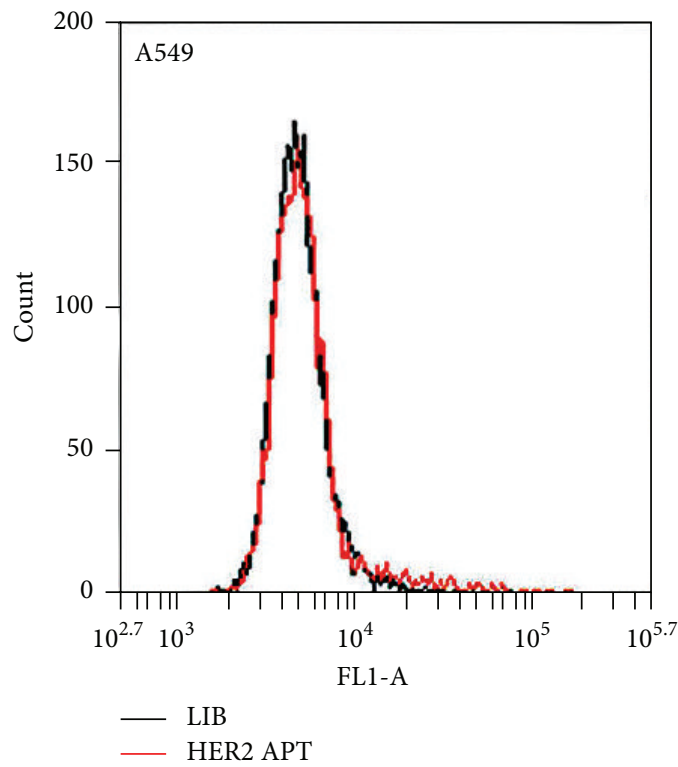

(c)

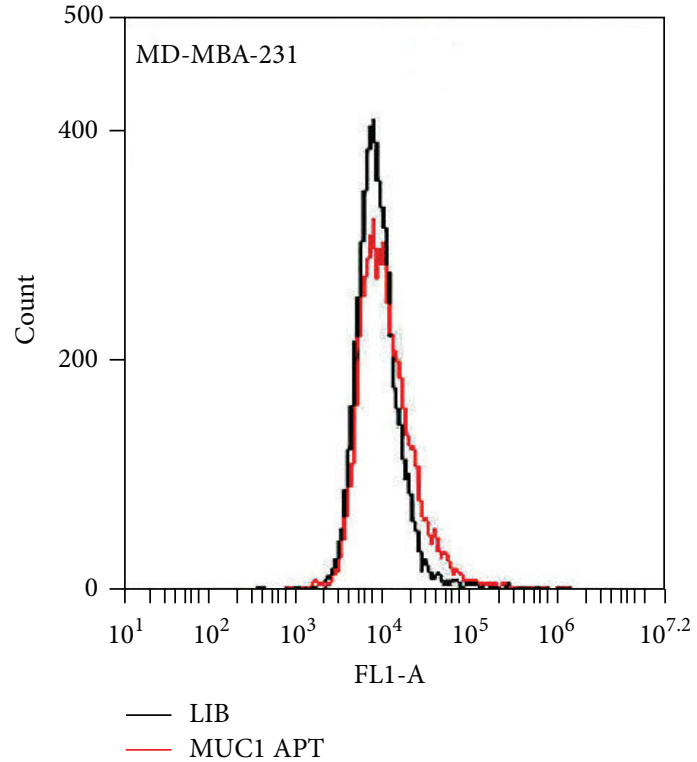

(b)

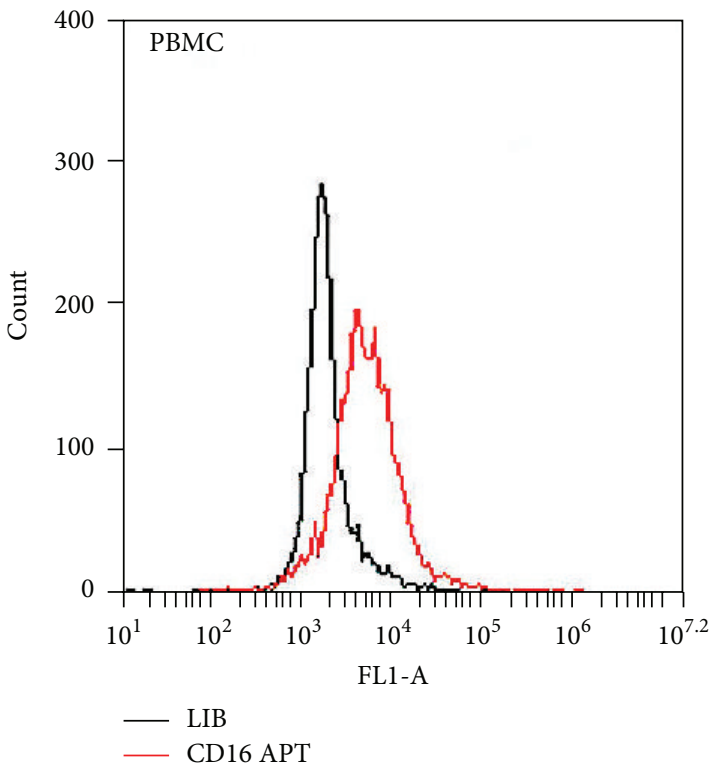

(d)

Figure 2: Evaluation of the binding affinity and specificity of the aptamers. FAM-labeled aptamers were incubated with cells and analyzed by flow cytometry (red lines). FAM-labeled random DNA (lib) was also incubated with the cells and served as the control (black lines). (a and b) Binding of MUC1 aptamer with the MUC1-positive A549 lung cancer cells and the MUC1-negative MDA-MB-231 cells, respectively. (c) Binding of the control HER2 aptamer with A549 cells. (d) Binding of the CD16 aptamer with PBMC.

3.5. Binding of Aptamer-Modified NPs to Target Cells. To evaluate whether MUC1 aptamer-modified NPs would attach to MUC1-positive cancer cells, these NPs were used to compete with free MUC1 aptamers for binding with A549 cells. Specifically, A549 cells were incubated with FAM-labeled MUC1 aptamers, in the presence or absence of the aptamer-modified NPs, and subjected to flow cytometry. The results showed that free aptamer's binding to target cells significantly decreased in the presence of aptamer-modified NPs (Figure 5(a)), indicating that these NPs also bound to A549 cells and competed with free aptamer for the binding sites. Notably, unmodified NPs failed to generate a competition effect.

To investigate whether CD16 aptamer-modified NPs would attach to PBMCs, similar competition experiments were conducted. Specifically, PBMC was incubated with FAM-labeled CD16 aptamers, in the presence or absence of the NPs functionalized with CD16 aptamers, and analyzed by flow cytometry. As shown in Figure 5(b), the binding of CD16 aptamer to PBMC was dramatically reduced in the presence of the aptamer-modified NPs, suggesting that these NPs 


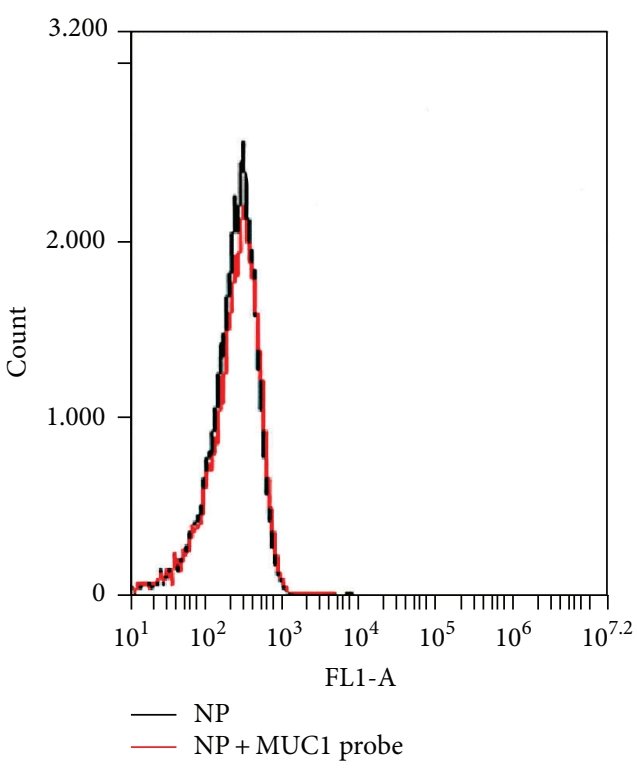

(a)

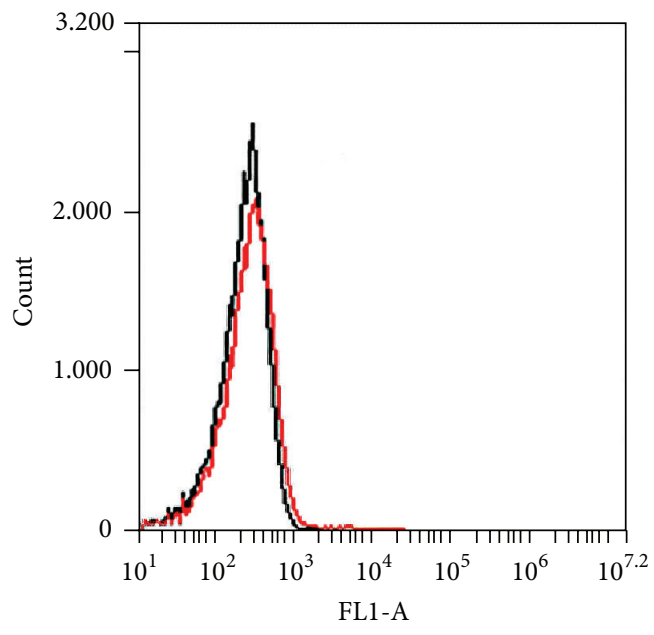

— $\mathrm{NP}$ CD16-NP + MUC1 probe

(c)

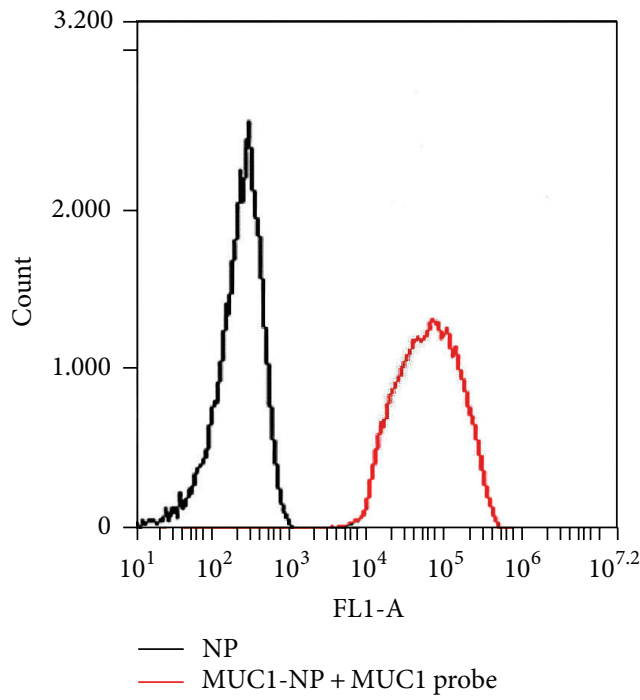

(e)

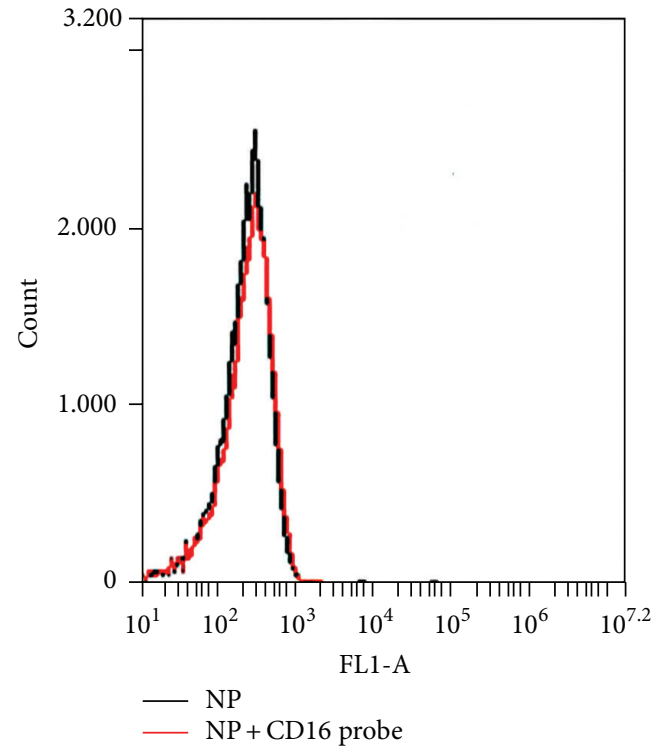

(b)

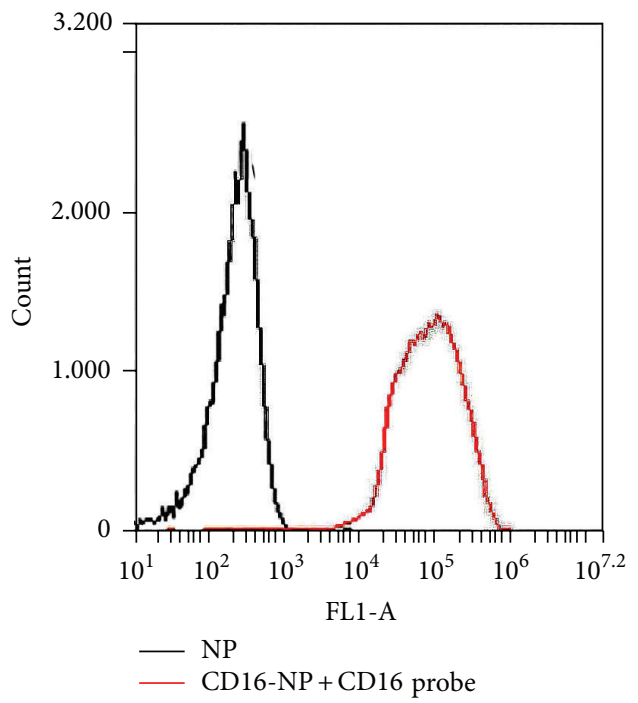

(d)

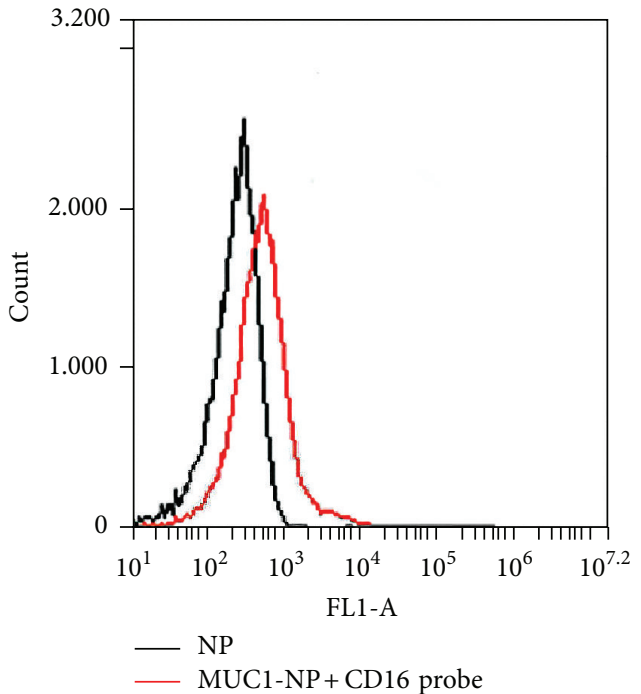

(f)

FIgURE 3: Continued. 


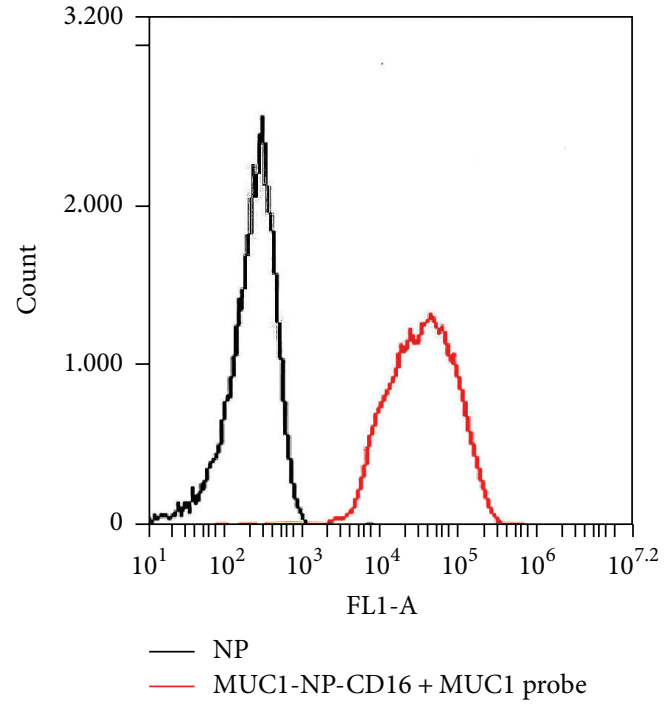

(g)

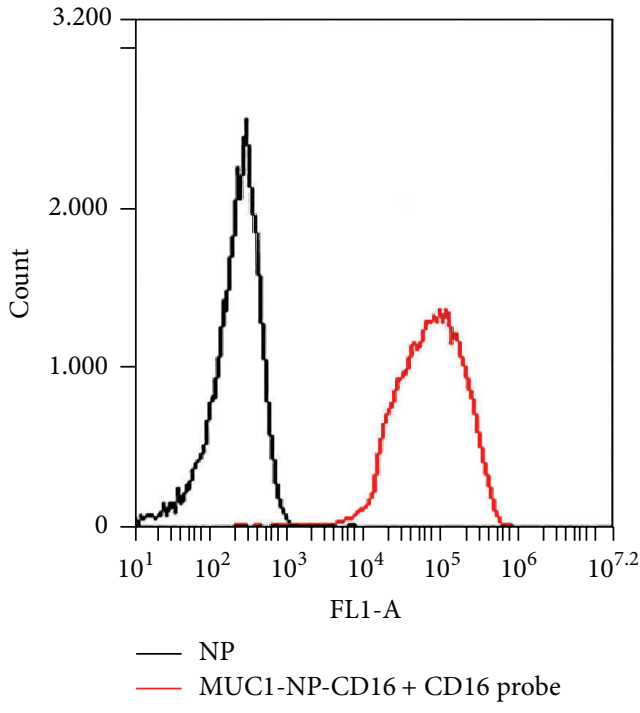

(h)

FIGURE 3: Evaluation of the conjugation of the aptamers with NP. MUC1 probe and CD16 probe were FAM-labeled DNA segments designed to hybridize with the MUC1 and the CD16 aptamers, respectively. Unmodified or aptamer-modified NPs were incubated with the probes and subjected to flow cytometry. Red lines represent signals from the beads treated with FAM-labeled DNA probe. Black lines represent the background signals from the untreated beads. (a) NP treated with MUC1 probe. (b) NP treated with CD16 probe. (c) CD16-NP treated with MUC1 probe. (d) CD16-NP treated with CD16 probe. (e) MUC1-NP treated with MUC1 probe. (f) MUC1-NP treated with CD16 probe. (g) CD16-NP-MUC1 treated with MUC1 probe. (h) CD16-NP-MUC1 treated with CD16 probe.

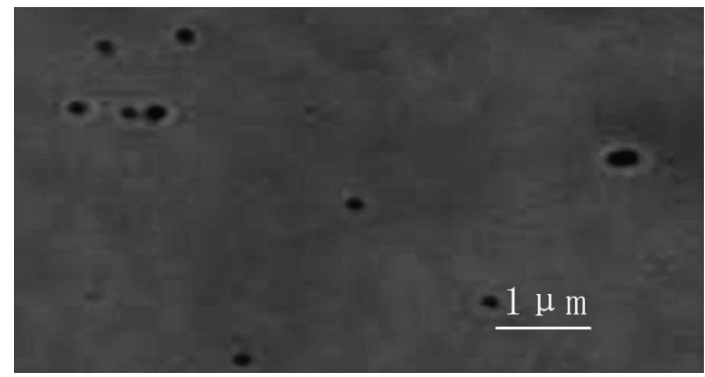

(a)

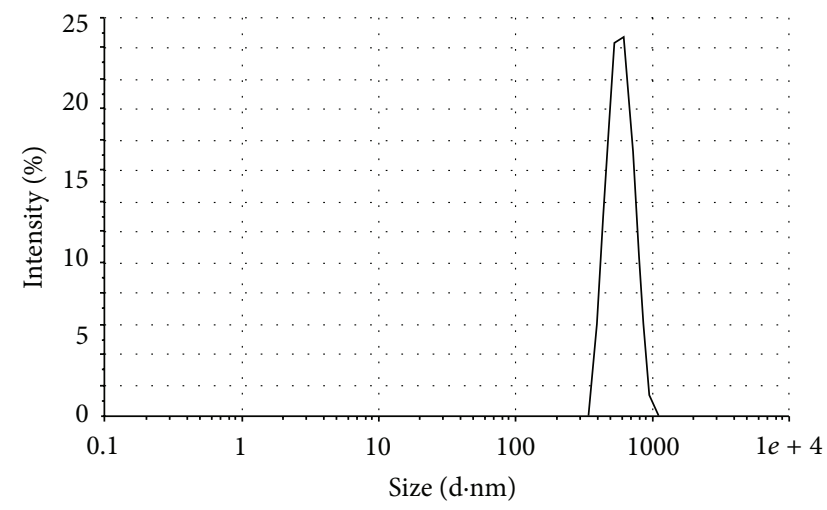

(c)

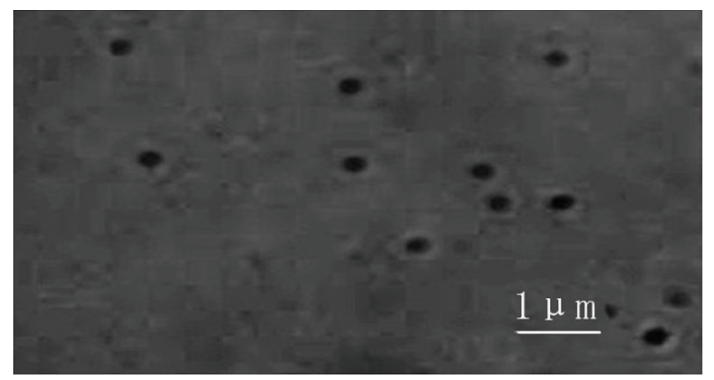

(b)

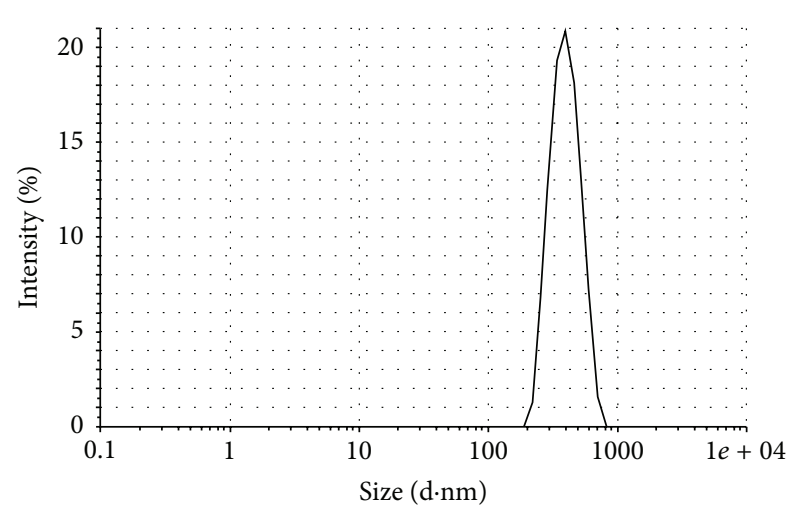

(d)

Figure 4: Morphological characterization of the amphipathic NP. (a and b) Confocal microscopic images showing the morphology of the amphipathic NP (a) and the unmodified NP (b). (c and d) DLS assay showing the size distributions of the amphipathic NP (c) and the unmodified NP (d). 


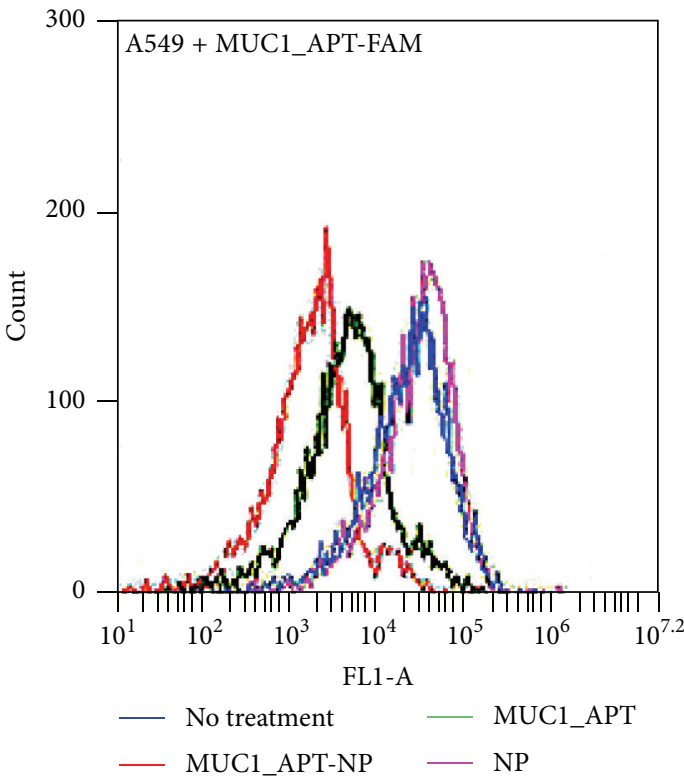

(a)

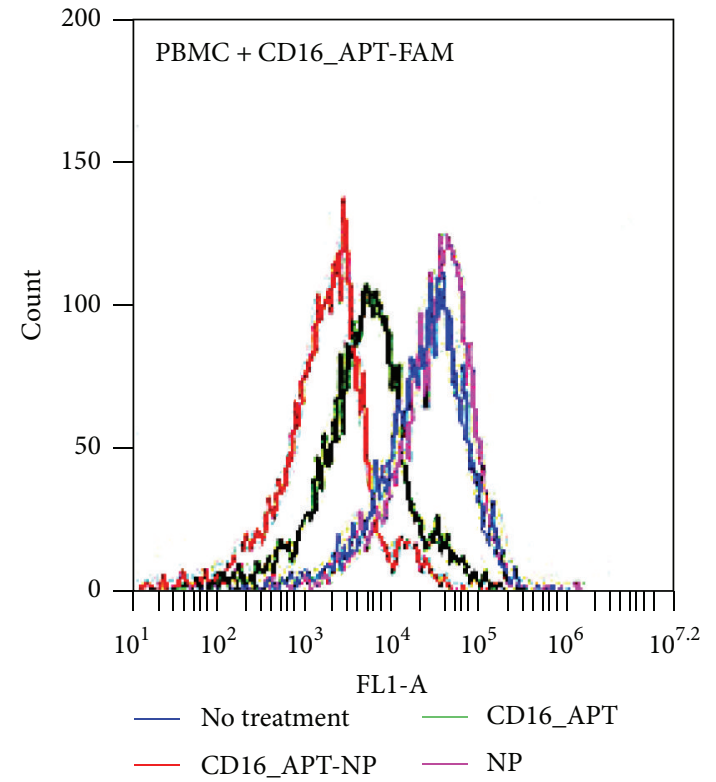

(b)

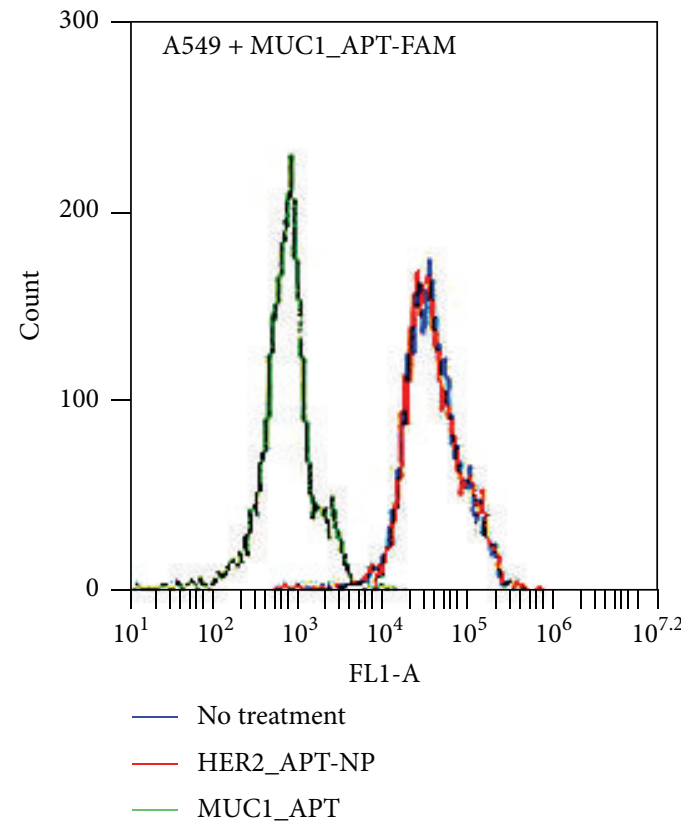

(c)

FIGURE 5: Evaluation of the binding of aptamer-modified NP to target cells by competition study. Cells were incubated with FAM-labeled aptamer. Free aptamer, aptamer-modified NP, or blank NP was added to compete for the binding sites on these cells, which were analyzed by flow cytometry. (a) A549 cells incubated with FAM-labeled MUC1 aptamer (blue line) and competed with MUC1-NP (red line), MUC1 aptamer (green line), or blank NP (purple line). (b) PBMC incubated with FAM-labeled CD16 aptamer (blue line) and competed with CD16NP (red line), CD16 aptamer (green line), and blank NP (purple line). (c) A549 cells incubated with FAM-labeled MUC1 aptamer (blue line) and competed with HER2-NP (red line) and MUCl aptamer (green line).

bound to PBMC and competed with CD16 aptamers for the binding sites. Unmodified NPs produced no competition effect.

The above results showed that NPs modified with MUC1 aptamer could attach to A549 cells. We wondered whether
NPs modified with any DNA (such as a HER2 aptamer) could also attach to MUC1-positive tumor cells. To address this issue, NPs modified with a HER2 aptamer were used to compete with FAM-labeled MUC1 aptamer for binding with A549 cells (Figure 5(c)). The results showed that HER2 


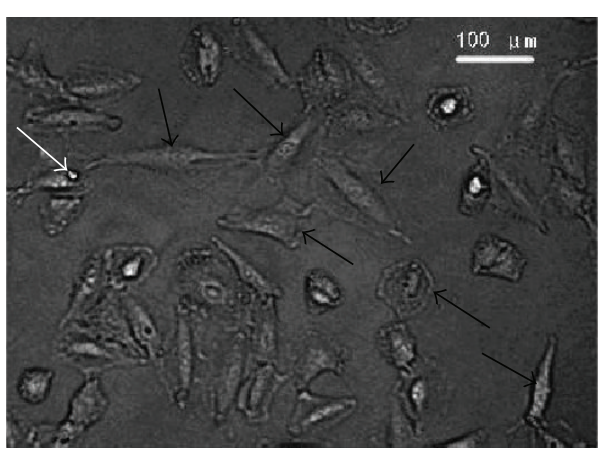

(a)

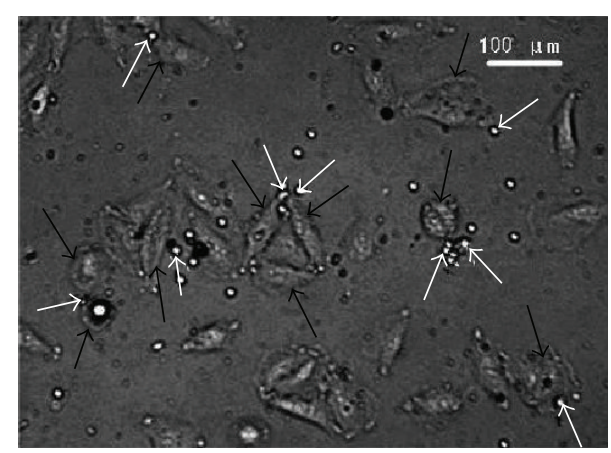

(b)

FIGURE 6: Effects of amphipathic NP on spatial relationship between NK and A549 cells. Live A549 cells were cocultured with fresh PBMC, treated with either the unmodified NP (a) or the amphipathic NP (b), washed, and evaluated by dark-field microscopy. Black arrows pointed to A549 cells, while white arrows pointed to PBMC.

aptamer-modified NPs did not reduce MUC1 aptamer's binding to A549 cells, indicating that these NPs did not bind with A549 cells.

3.6. Amphipathic NPs Recruited NK to A549 Cells. The above results showed that aptamer-modification facilitated the binding of NPs to A549 and NK cells, respectively. However, it was still unknown whether NP modified with both CD16 and MUC1 aptamers (the amphipathic NP) could attach to both kinds of cells and bring them close to each other. To answer this question, blank NP or the amphipathic NP was separately added to a mixture of NK and A549 cells. After unattached NK cells were removed by wash, the remaining cells were analyzed by dark-field microscopy. As shown in Figure 6, after treatment with blank NP, few NK cells remained in the culture dish, whereas after treatment with amphipathic NP, much more NK cells were still attached to A549 cells. The results indicated that the amphipathic NP could recruit more NK to the vicinity of A549 cells.

3.7. NK Cytotoxicity to MUC1-Positive Cancer Cells Was Enhanced by Amphipathic NP. The above study indicated that the amphipathic NP could recruit more NK to MUC1-positive A549 tumor cells. However, it was unknown whether the NK reaction to these tumor cells would also be enhanced. To address this issue, NK cytotoxicity studies were conducted. A549 cells were cocultured with PBMCs and treated separately with blank NP, amphipathic NP (MUC1-NP-CD16), a mixture of free CD16 and MUC1 aptamers (MUC1 APT + CD16 APT), and NP modified with CD16 and HER2 aptamers (HER2-NP-CD16). The NK cytotoxicity to A549 cells for each study group was analyzed by MTS assay. As illustrated in Figure 7(a), the amphipathic NP significantly enhanced NK cytotoxicity against A549 cancer cells, while blank NP, free aptamers, or HER2-CD16-NP failed to generate an obvious effect. The results indicated that pulling together NK and A549 cells by the amphipathic NP could activate NK to react against the MUC1-positive cancer cells.

To further investigate whether the NK antitumor reaction induced by the amphipathic NP was targeted towards the MUC1-positive cancer cells, similar cytotoxicity study was also conducted with the MUC1-negative MD-MBA-231 cells. Specifically, MD-MBA-231 cells were cocultured with PBMC, treated with amphipathic NP, washed, and analyzed by MTS assay for viability. As shown in Figure 7(b), the amphipathic NP failed to enhance the NK reaction against these MUC1negative cells. Taken together, the above data indicated that the NK cytotoxicity enhanced by the amphipathic NP was targeted towards the MUCl-positive cancer cells, but not the MUC1-negative control cells.

\section{Discussion}

The primary purpose of this study was to construct an amphipathic nanoparticle that was modified with CD16 and MUC1 aptamers, which were implanted onto the particle via biotinstreptavidine reaction (Figure 1). The MUC1 aptamer was verified to bind with the MUC1-positive A549 lung cancer cells, but not the MUC1-negative MDA-MB-231 cells (Figures 2(a) and 2(b)). Similarly, the CD16 aptamer was also confirmed to bind with the PBMC that was rich in CD16-positive NK cells (Figure 2(d)). DNA hybridization experiments showed that the aptamers were indeed connected to the nanoparticles (Figure 3). The average size of the aptamer-modified NPs was about $574 \mathrm{~nm}$, with a single-peak size distribution (Figure 4). Competition assays indicated that aptamer-modified NPs could bind with both the MUC1-positive cancer cells and CD16-positive immune cells (Figure 5). Moreover, a significantly increased number of NKs were observed to attach to the MUC1-positive cancer cells in the presence of amphipathic NPs (Figure 6), suggesting that the nanostructure could pull the NK close to the target cancer cells. In addition, this nanoparticle significantly enhanced the NK's antitumor immune cytotoxicity to the MUC1-positive A549 cancer cells (Figure 7(a)), but not that to the MUC1-negative MD-MBA231 control cells (Figure 7(b)), indicating that a targeted NK reaction against the MUC1-positive tumor cells was promoted by the amphipathic NPs.

MUC1 is an important molecular target for cancer treatment. It is underexpressed in normal tissue [13] but overexpressed in majority of adenocarcinomas, including lung, 


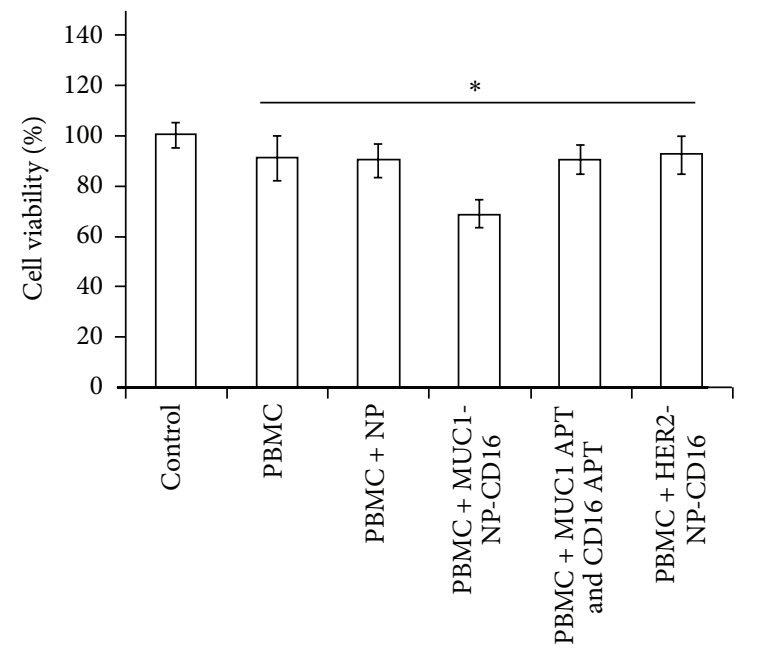

(a)

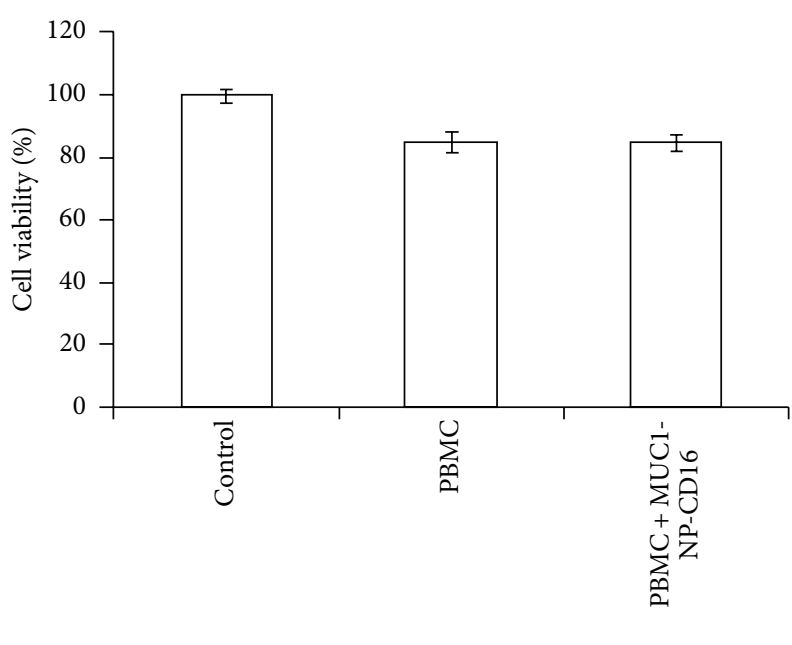

(b)

FIgURE 7: Modulation of the NK cytotoxicity to cancer cells by amphipathic NP. The cancer cells were cocultured with PBMC in the presence of various treatments and analyzed for viability. (a) MUC1-positive A549 cells treated with blank NP, the amphipathic NP (MUC1-NP-CD16), a mixture of free CD16 and MUC1 aptamers, and NP modified with HER2 and CD16 aptamers (HER2-NP-CD16). (b) MUC1-negative MDMBA-231 cells treated with the amphipathic NP. The NK cytotoxicity was evaluated by a standard MTS assay per manufacturer's protocol (mean $\pm \mathrm{SD}, n=8)$. The star indicates a statistically significant difference $(p<0.01)$.

liver, breast [14], ovarian, colon, pancreatic, prostate cancers, and multiple myeloma [15-17]. The overexpression of MUC1 mucin may be associated with cancer progression and metastasis of many malignancies [18-20]. Because MUC1 is overexpressed in many types of adenocarcinoma and underexpressed in normal cells, it is considered as an ideal molecular target for cancer therapy. As a result, several attempts for development of MUC1-targeted cancer therapy have been reported, including targeted drug delivery to MUC1-positive tumor cells with aptamer-guided vehicles [21], tumor vaccine based on MUC1 protein for inducing anticancer $\mathrm{T}$ cell response [5], and MUC1-targeted photodynamic cancer therapy with photosensitizer carried by MUC1 aptamers [22]. So far, however, there is no report in the literature on directing NK cells to MUC1-positive tumor cells for enhancement of the NK reaction against these cancer cells. In this study, we built an aptamer-based amphipathic nanoparticle that could recruit NK cells to MUC1-positive cancer cells. We found that the nanoparticle could induce a targeted NK cytotoxicity to MUC1-positive tumor cells. The results suggested that, in addition to anticancer drugs, $\mathrm{T}$ lymphocytes, or photodynamic energy, NK cells might also be recruited to develop a novel targeted therapy against MUC1-positive tumors.

The mechanism by which the amphipathic NP promotes the NK reaction to MUC1-positive cancer cells is unclear at present. We suspect that the enhanced NK cytotoxicity to tumor cells may be related to increased interaction between the two types of cells. Our data indicate that the amphipathic NP can recruit NK to the vicinity of MUC1-positive tumor cells (Figure 6). This will probably increase the chance of interaction between the NK and the target cancer cells, and, through some unknown molecular mechanisms, activate the NK to initiate an anticancer reaction. Similarly, a previously study also reported that recruiting NK cells to the neighborhood of HGF-R positive tumor cells could enhance the NK reaction against these cancer cells [9]. However, the detailed molecular mechanisms of the NK activation for increased anticancer response remain to be unveiled. In any case, this study found that pulling together NK and MUC1-positive tumor cells could enhance the NK reaction against the A549 adenocarcinoma cells. This finding may lead to the development of novel targeted therapy against MUC1-positive tumors through recruitment of $\mathrm{NK}$ cells for anticancer reaction. Nevertheless, it should be noted that the current study is just an early exploration of recruiting NK to fight against MUC1expressing cancer cells. In order to further develop and implement the strategy, extensive future research is still warranted, including studies on development of nuclease-resistant aptamers, selection of optimal nanoparticles, exploration of proper methods to connect the aptamers with the nanostructure, and evaluation of in vivo therapeutic efficacy with animal studies.

In summary, a novel amphipathic nanoparticle was constructed by implanting CD16 and MUC1 aptamers onto its surface. This nanoparticle pulled NK (or other CD16-positive immune cells) and MUC1-positive cancer cells together in vitro and significantly enhanced the $\mathrm{NK}$ cytotoxicity to MUC1-positive cancer cells. The results suggest that NK cells may be mobilized to target against MUC1-positive tumor cells.

\section{Conflict of Interests}

The authors declare that there is no conflict of interests regarding the publication of this paper. 


\section{Acknowledgments}

The authors acknowledge the funding support from the Chinese Ministry of Science and Technology (2011CB933504) and the 111 Project from the Chinese Ministry of Education.

\section{References}

[1] A. Vermes, H.-J. Guchelaar, and J. Dankert, "Flucytosine: a review of its pharmacology, clinical indications, pharmacokinetics, toxicity and drug interactions," Journal of Antimicrobial Chemotherapy, vol. 46, no. 2, pp. 171-179, 2000.

[2] T. Reuther, T. Schuster, U. Mende, and A. C. Kübler, "Osteoradionecrosis of the jaws as a side effect of radiotherapy of head and neck tumour patients-a report of a thirty year retrospective review," International Journal of Oral and Maxillofacial Surgery, vol. 32, no. 3, pp. 289-295, 2003.

[3] S. A. Rosenberg, "Progress in human tumour immunology and immunotherapy," Nature, vol. 411, no. 6835, pp. 380-384, 2001.

[4] K. Tarte and B. Klein, "Dendritic cell-based vaccine: a promising approach for cancer immunotherapy," Leukemia, vol. 13, no. 5, pp. 653-663, 1999.

[5] R. Kovjazin, I. Volovitz, Y. Kundel et al., "ImMucin: a novel therapeutic vaccine with promiscuous MHC binding for the treatment of MUC1-expressing tumors," Vaccine, vol. 29, no. 2930, pp. 4676-4686, 2011.

[6] D. Berd, T. Sato, H. C. Maguire Jr., J. Kairys, and M. J. Mastrangelo, "Immunopharmacologic analysis of an autologous, hapten-modified human melanoma vaccine," Journal of Clinical Oncology, vol. 22, no. 3, pp. 403-415, 2004.

[7] J. S. Yu, G. T. Liu, H. Ying, W. H. Yong, K. L. Black, and C. J. Wheeler, "Vaccination with tumor lysate-pulsed dendritic cells elicits antigen-specific, cytotoxic T-cells in patients with malignant glioma," Cancer Research, vol. 64, no. 14, pp. 49734979, 2004.

[8] C. Mesa and L. E. Fernández, "Challenges facing adjuvants for cancer immunotherapy," Immunology and Cell Biology, vol. 82, no. 6, pp. 644-650, 2004.

[9] A. Boltz, B. Piater, L. Toleikis, R. Guenther, H. Kolmar, and B. Hock, "Bi-specific aptamers mediating tumor cell lysis," The Journal of Biological Chemistry, vol. 286, no. 24, pp. 2189621905, 2011.

[10] Y. Hu, J. Duan, Q. Zhan, F. Wang, X. Lu, and X.-D. Yang, "Novel MUC1 aptamer selectively delivers cytotoxic agent to cancer cells in vitro," PLoS ONE, vol. 7, no. 2, Article ID e31970, 2012.

[11] J. Taylor-Papadimitriou, J. Burchell, D. W. Miles, and M. Dalziel, "MUC1 and cancer," Biochimica et Biophysica Acta-Molecular Basis of Disease, vol. 1455, no. 2-3, pp. 301-313, 1999.

[12] P. Zhang, N. X. Zhao, Z. H. Zeng et al., "Using an RNA aptamer probe for flow cytometry detection of CD30-expressing lymphoma cells," Laboratory Investigation, vol. 89, no. 12, pp. 14231432, 2009.

[13] P. Chaturvedi, A. P. Singh, and S. K. Batra, "Structure, evolution, and biology of the MUC4 mucin," The FASEB Journal, vol. 22, no. 4, pp. 966-981, 2008.

[14] E. A. Rakha, R. W. G. Boyce, D. A. El-Rehim et al., "Expression of mucins (MUC1, MUC2, MUC3, MUC4, MUC5AC and MUC6) and their prognostic significance in human breast cancer," Modern Pathology, vol. 18, no. 10, pp. 1295-1304, 2005.

[15] K. Ohuchida, K. Mizumoto, D. Yamada et al., "Quantitative analysis of $\mathrm{MUC1}$ and MUC5AC mRNA in pancreatic juice for preoperative diagnosis of pancreatic cancer," International Journal of Cancer, vol. 118, no. 2, pp. 405-411, 2006.

[16] S. C. Chauhan, N. Vinayek, D. M. Maher et al., "Combined staining of TAG-72, MUC1, and CA125 improves labeling sensitivity in ovarian cancer: antigens for multi-targeted antibodyguided therapy," Journal of Histochemistry \& Cytochemistry, vol. 55, no. 8, pp. 867-875, 2007.

[17] J. C. O'Connor, J. Julian, S. D. Lim, and D. D. Carson, "MUC1 expression in human prostate cancer cell lines and primary tumors," Prostate Cancer and Prostatic Diseases, vol. 8, no. 1, pp. 36-44, 2005.

[18] Y. S. Kim, J. Gum Jr., and I. Brockhausen, "Mucin glycoproteins in neoplasia," Glycoconjugate Journal, vol. 13, no. 5, pp. 693-707, 1996.

[19] M. Sachdeva and Y.-Y. Mo, "MicroRNA-145 suppresses cell invasion and metastasis by directly targeting mucin 1," Cancer Research, vol. 70, no. 1, pp. 378-387, 2010.

[20] S. Satoh, Y. Hinoda, T. Hayashi, M. D. Burdick, K. Imai, and M. A. Hollingsworth, "Enhancement of metastatic properties of pancreatic cancer cells by MUC1 gene encoding an antiadhesion molecule," International Journal of Cancer, vol. 88, no. 4, pp. 507-518, 2000.

[21] C. Yu, Y. Hu, J. Duan et al., "Novel aptamer-nanoparticle bioconjugates enhances delivery of anticancer drug to MUC1-positive cancer cells in vitro," PLoS ONE, vol. 6, no. 9, Article ID e24077, 2011.

[22] C. S. M. Ferreira, M. C. Cheung, S. Missailidis, S. Bisland, and J. Gariépy, "Phototoxic aptamers selectively enter and kill epithelial cancer cells," Nucleic Acids Research, vol. 37, no. 3, pp. 866-876, 2009. 

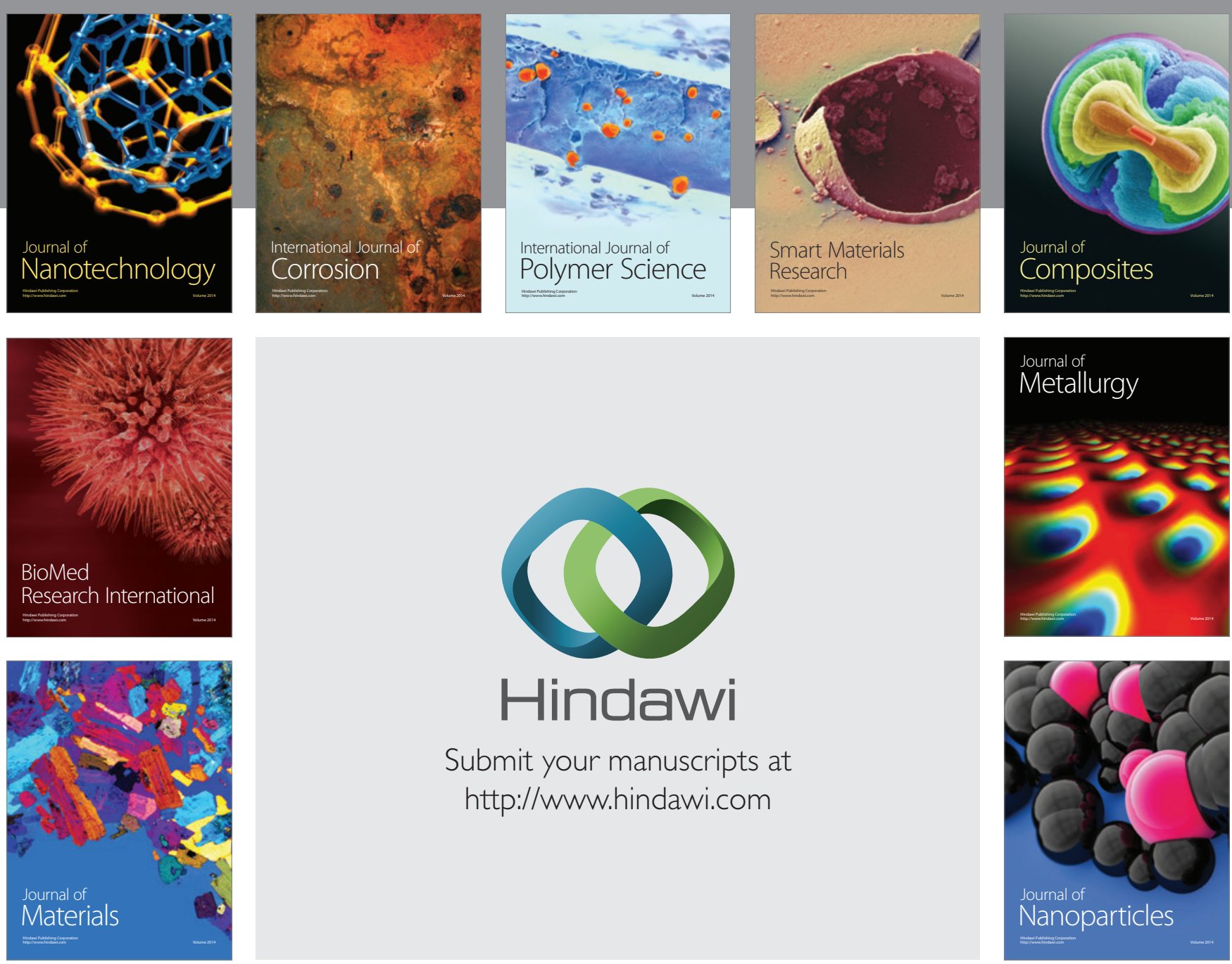

Submit your manuscripts at http://www.hindawi.com
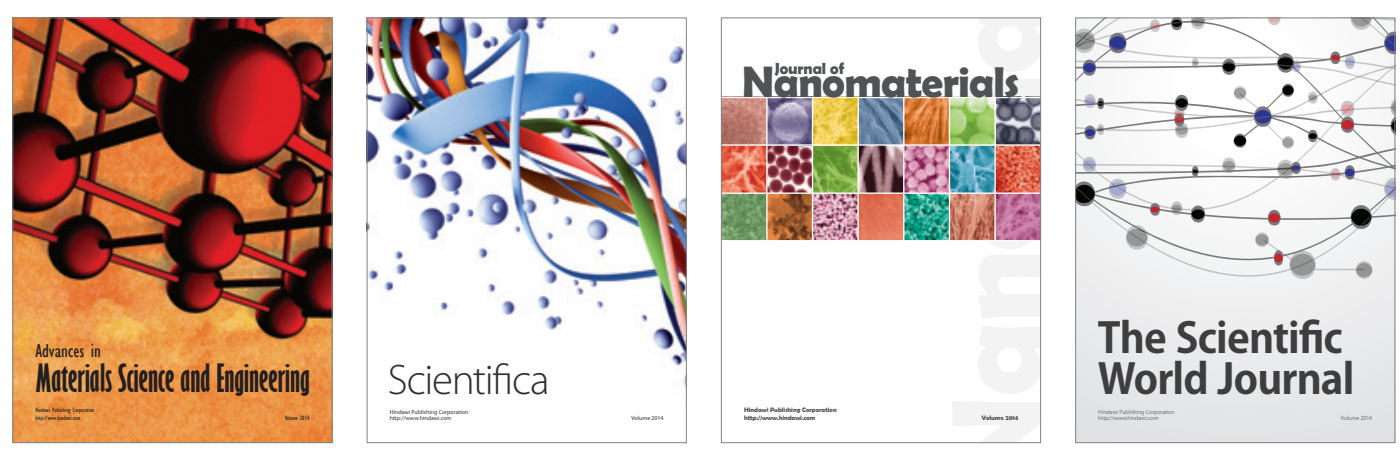

\section{The Scientific World Journal}
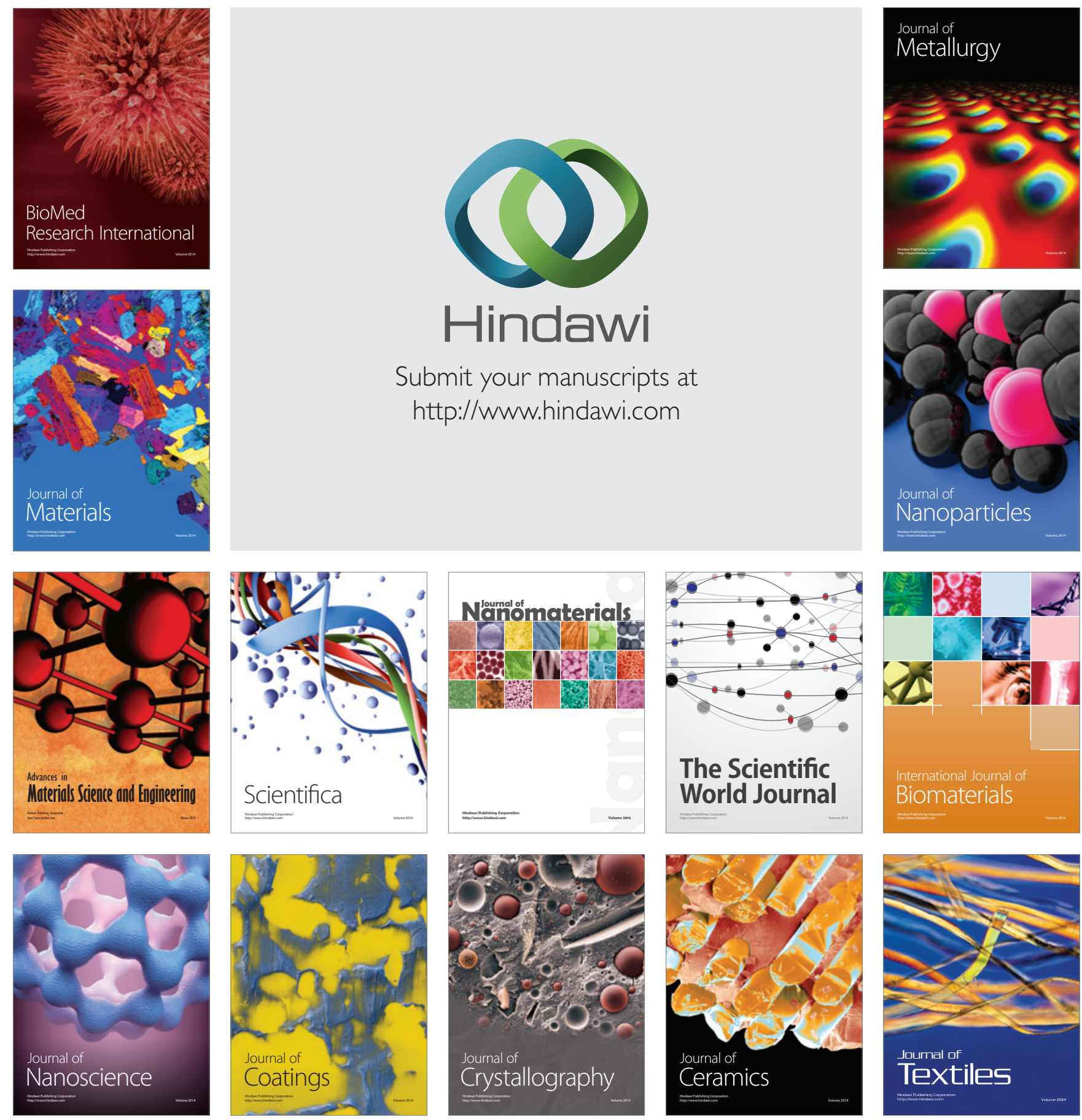